\title{
Criterios \\ Transformaciones y cambios del seminario de San Telmo durante la segunda mitad del siglo XVIII
}

Carlos Sambricio Rivera-Echegaray

Catedrático de Historia de la Arquitectura y del Urbanismo. Universidad Politécnica de Madrid

\section{Resumen}

Los últimos años del siglo XVIII deciden inexorablemente nuevas formas para el Seminario de San Telmo, en parte provocadas por un resurgimiento económico, en parte debido a las politicas de embellecimiento de Olavide en Sevilla, entre las que se encontraban varias actuaciones estrechamente vinculadas a San Telmo. Paralelamente, los debates en la ciudad acerca de la forma rectangular de la planta del edificio protagonizaron esta etapa histórica y constructiva del inmueble.

\section{Palabras clave}

\section{Real Colegio Seminario de San Telmo}

Patrimonio inmueble

$1770-1800$

Historia

Sevilla

Intervención
El gran momento en la construcción del Seminario de San Telmo fue, sin duda, el último cuarto del siglo XVIII, cuando tres factores, en principio independientes entre sí (el resurgimiento económico del Seminario; la voluntad -por los pleitos con el Ayuntamiento- en concluir las obras y, por último, la política de embellecimiento llevada a cabo por Olavide en Sevilla) afectaron al plan de obras del Seminario.

Iniciadas las obras de San Telmo a finales del XVII, un siglo más tarde estas no estaban ni remotamente concluidas. Puesta en 1681 la primera piedra, en 1698 se declaraba haberse concluido los dos dormitorios, el Refectorio, la sastrería, cocina, cuarto del cocinero y granero, secadero de invierno y lavadero. Tras lo cual las obras (en un edificio capaz en un principio de albergar a 50 muchachos y luego, a finales del XVIII, de hospedar a casi 200) quedaron paralizadas. Tuvieron que pasar casi treinta años hasta que se encomendara a Figueroa la construcción de la Iglesia, y aquel encargo se demostró clave en el conjunto de la obra por cuanto que, como señaló Herrera García ...comenzada la obra en septiembre de dicho año (1723) expresó Figueroa la conveniencia de unir la iglesia al resto de la edificación, pues construida aquella en el centro de la traza de esta, quedaba aislada de lo edificado anteriormente en el ala del río. La Diputación, en vista de aqueIlo, acordó hacer un Claustro y una Enfermería, según diseño de Matías de Figueroa, hijo de Leonardo, que venia trabajando con él.

Retengamos la idea: construida aquella en el centro de la traza de esta, quedaba aislada de lo edificado anteriormente en el ala del río. Edificada en el centro de la traza originaria, sucedía sin embargo que quedaba aislada de lo existente, por cuanto que desde 1698 el Seminario se había reducido a un espacio menor, del que no tenemos documentación gráfica. Por ello la propuesta de Figueroa tuvo un alcance superior al mero proyecto de edificación de una Iglesia al proponer que esta quedara unida al resto de la edificación. Lo singular de la idea fue definir un eje compuesto como sucesión de piezas (fachada principal, Claustro del edificio e Iglesia) buscando que dicho eje (el único existente, por cuanto solo en el siglo XX Basterra propondría, erráticamente, un eje transversal que nunca hasta entonces había existido) se entendiera como eje de composición, reclamando continuar la parte inconclusa y edificando la inexistente ala simétrica situada entre el Eje y las puertas Nueva y de Jerez.

Entre 1724, fecha en que se bendijo la Iglesia, y 1734, en que se concluyó la fachada principal, en el Seminario se ejecutaron un conjunto de obras determinantes en la traza definitiva del edificio. Puesto que sólo estaba realizada la parte del Seminario que daba al río, Figueroa condicionó la imagen exterior del conjunto al obligar que lo no construido se trazara desde los criterios compositivos establecidos en la parte existente, marcando en consecuencia el futuro del edificio.

Habrá quien opte por valorar -en la propuesta de Figueroa- sólo desde el valor de cada una de las piezas trazadas (Iglesia, Sacris- 


\section{$066-067$ \\ Criterios}

Transformaciones y cambios de San Telmo durante la segunda mitad del siglo XVIII

\section{PH51 - Diciembre 2004}

tía o Claustro) independizando la "máscara barroca" que es la fachada del resto del proyecto. Afirmo esto convencido que eruditos celosos del valor del documento argumentaran que cada una de estas piezas son independientes entre sí, por cuanto fueron ejecutadas por distintas manos; y estoy convencido que aceptaran lo que he señalado sólo por cuanto canto las excelencias de un artífice local, pero no porque compartan mi idea sobre cuanto lo excepcional de la idea de Figueroa fue buscar la conclusión del edificio carente, hasta el momento, de imagen urbana. Reitero, en consecuencia, que el proyecto de Figueroa fue una propuesta que iba mucho mas allá que el mero trazado de una iglesia, y ello por dos motivos: primero, porque al definir un eje compositivo constituido por tres piezas (independientes en su función, si bien articuladas entre si) proponía una fachada para el edificio, rematando lo todavía no existente; en segundo lugar, porque al utilizar sabiamente los recursos del lenguaje arquitectónico daba unidad al edificio (confundiendo voluntariamente lo existente con lo propuesto) proponiendo en consecuencia una imagen urbana y convirtiendo San Telmo en referencia arquitectónica cuando la Fábrica de Tabacos ni siquiera estaba proyectada. Entender, pues, la Fachada desde el valor de su escultura decorativa o la Iglesia, desde el mérito del Retablo, sería empobrecer un proyecto arquitectónico compuesto por tres piezas, cada una de las cuales tuvo (en su traza) el valor añadido de formar parte del todo que fue el eje principal, posibilitando en consecuencia retomar la imprecisa traza originaria, aquella de la que los documentos encontrados sólo apuntan "debía ser cuadrilonga".

Finalizadas las obras trazadas por los Figueroa, las obras se interrumpieron de nuevo hasta avanzada la segunda mitad del siglo, cuando en torno a 1760 se produjo una singular triple circunstancia: se produjo el resurgir económico del Seminario; Olavide formuló su propuesta de embellecimiento de Sevilla y, buscando el Ayuntamiento apropiarse del terreno no construido de San Telmo (y temerosa su Diputación que pudiera conseguirlo) se decidió (tras décadas de inactividad edilicia) concluir
1. Apeaderos en la zona norte del edificio, de Juan Talavera de la Vega. Foto Eugenio Fernández Ruiz

2. Salón principal del Palacio de San Telmo en la época de los Montpensier, actual salón de recepciones de la Consejería de Presidencia, rehabilitado por Vázquez Consuegra en 1992. Foto José Manuel Santos Madrid las obras, debiendo para ello cimentar, como primera medida, el perímetro original del proyecto.

El resurgimiento económico del Seminario fue estudiado por Herrera García, quien comentó cómo ....ante la petición del Gobierno de transvasar el caudal sobrante, se reclamó continuar las obras, pidiendo permiso para gastar anualmente en ella cuatro mil pesos. Y la petición queda en suspenso ante la propuesta del Cabildo de Sevilla de iniciar las obras del Camino Real. Convendría, obviamente, disponer de un estudio económico que explicitase qué suponía, en aquel momento, el gasto anual de cuatro mil pesos: retengamos, entre tanto, la noticia y comentemos qué fueron las propuestas de Olavide para Sevilla, obras de embellecimiento que cabría comparar con las dispuestas por Carlos III para Madrid, con las ejecutadas por Ricla en Barcelona, con los proyectos de Arenal en Bilbao, de Alameda en Málaga, de Campo Grande en Valladolid, de Espolón en Burgos... y que claramente asustaron a la Diputación del Seminario (por las consecuencias que pudieron tener) forzando reactivar la propuesta de Figueroa.

La política de embellecimiento llevada a término en las ciudades europeas de aquellos años ha sido bien estudiada y conocemos cuáles fueron los propósitos de aquel devoir d'embellir definido en la Encyclopedie de Diderot y d'Alambert. "Embellecer" no sólo consistió en introducir la naturaleza en la ciudad, sustituyendo los barrocos espacios cerrados de las plazas mayores por paseos y alamedas sino que buscó, simultáneamente, desarrollar cuatro tipos de actuación: en primer lugar, valorar el edificio oficial como pieza del Poder, romper su situación de pieza construida entre medianeras y presentarlo como edificio con cuatro fachadas: y el proyecto de Marquet/Ventura Rodriguez para la madrileña Casa de Correos podría ser ejemplo, frente al todavía barroco edificio de Sabatini de la madrileña calle de Alcalá, actual Ministerio de Hacienda; en segundo lugar, se propuso la construcción de las hasta entonces inexistentes infraestructuras, iniciándose obras de alcantarillado, alumbrado y empedrado;
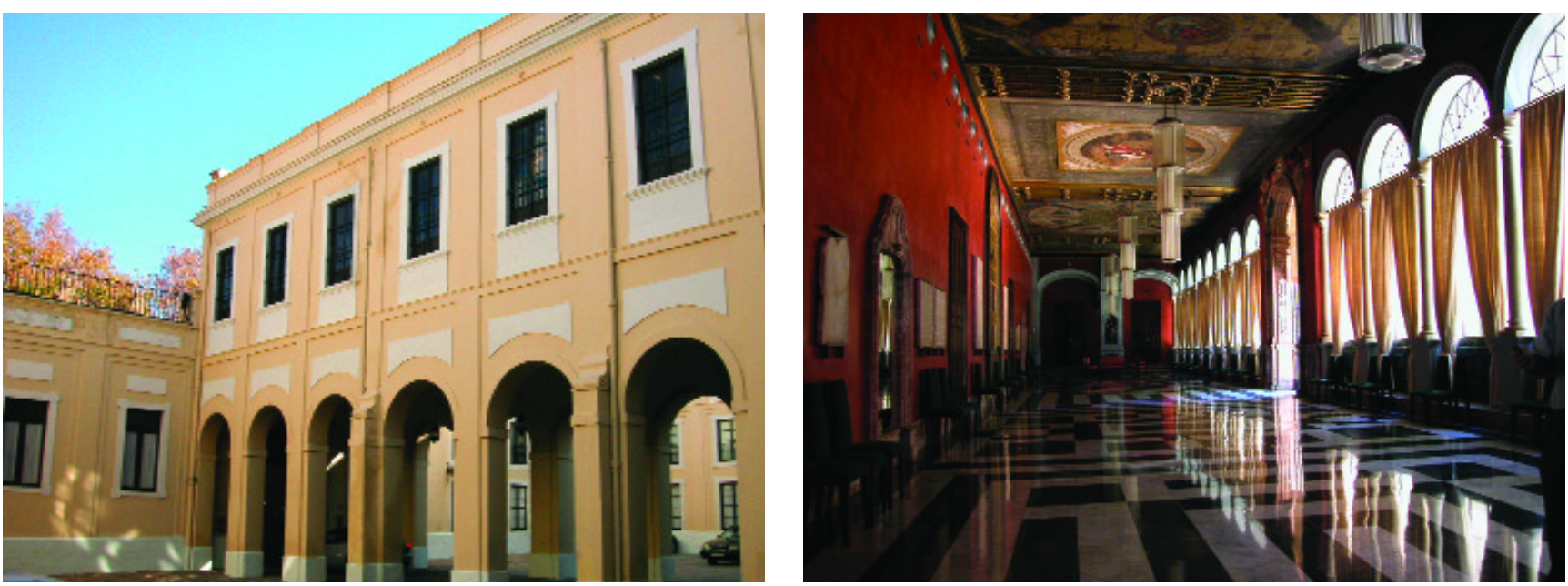
también creó conciencia sobre la necesaria alineación de las caIles, buscando que una misma vía tuviese idéntico ancho en todo su recorrido. Y, por último (y este es el tema que ahora mas nos interesa) actuó en el límite de la ciudad, tanto al sustituir los pedregosos y polvorientos caminos por alamedas arboladas como al abordar el tema de los espacios residuales extramuros o la embocadura de las alamedas en calles, resolviendo el tránsito de unas a otras mediante ornamentación consistente en puertas de entrada, jardines o símbolos emblemáticos tales como obelisco, fuentes...

Conocemos bien las reformas que Olavide propuso para Sevilla: reivindicó el ornato y embellecimiento de la ciudad mediante el adecentamiento de las márgenes del río; construyó paseos extramuros y decoró los accesos a la ciudad, facilitando al sevillano el disfrute de áreas hasta entonces consideradas como extramuros. Coherente con tales actuaciones, propuso la construcción del Puente nuevo entre Triana y Plaza de Armas, auspició el trazado del paseo que uniría Puerta de Triana con Puerta Real; se ordenaron las márgenes del río entre la Plaza de Santiago de la Espada hasta Puerta de San Juan y se urbanizó el entorno de Puerta Nueva. Pero quien estudie detenidamente el plano de Olavide observará cuatro actuaciones relacionadas con San Telmo que afectaron las obras del Seminario: adecuar el acceso a San Diego desde Sevilla; dar ingreso a la Fábrica de Tabacos desde la ciudad; adecentar y embellecer el espacio existente entre el Seminario y Torre del Oro y, último, actuar en el entorno de Puerta de Jerez y Puerta Nueva.

Olavide no sólo buscó introducir la naturaleza en ciudad sino que remodeló el perímetro de la población, ordenándolo: en una SeviIla sin apenas jardines interiores (las contadas huertas se situaban en las inmediaciones de la Iglesia de Santiago de la Espada o en el entorno de la Puerta de la Barqueta) y donde junto a San Telmo todavía existía el muelle de descarga (junto a San Diego estaba el Quemadero) su propuesta buscó potenciar el pequeño camino que marcaba el límite de la propiedad del suelo de la ciudad. Si junto a San Diego el Camino Real se había ya transformado en vía urbana y la embocadura del uno en otro se resolvió mediante una alameda de dos hilos (la misma que existía frente a la Fábrica de Tabacos) al llegar a San Telmo el ancho de este camino debía, ineludiblemente, reducir su amplitud al atravesar una propiedad que, desde hacia más de un siglo, anunciaba la conclusión de las obras del Seminario sin nunca iniciar las mismas.

Desde la lógica de quien actúa en la ciudad se forzó la ocupación de aquel suelo: pero fue entonces cuando un San Telmo -con nuevas posibilidades económicas- reaccionó pidiendo reiniciar las obras de la parte septentrional (la más próxima a la ciudad) alegando estar cimentada dicha zona y encontrarse en vías de ejecución la propuesta definida por Figueroa.

Para conocer el estado en que se encontraba la fábrica de San Telmo entre 1770 y 1800 contamos con una significativa docu- mentación gráfica, generada en su mayor parte como consecuencia de los pleitos que enfrentaron al Ayuntamiento con la Diputación del Seminario. Al contrastar unos con otros sorprende ver como la información que facilitan difiere, consecuencia sin duda de haberse levantado a instancias de unos o por encargos de otros: así, en planos fechados y datados con sólo una semana de diferencia (encargados unos por el Ayuntamiento, otros por la Diputación del Seminario) detalles aparentemente de segundo orden (como por ejemplo la existencia o no de determinada edificación dentro del conjunto) se destaca en el primero y se ignora en el segundo. De cualquier forma, y pese a lo subjetivo y arbitrario de la documentación gráfica conocida, la misma es útil por cuanto permite comprender qué sucedía más allá del recinto del Seminario, en su entorno inmediato. Gracias a los mismos conocemos los cambios urbanos que se produjeron en el entorno de la fábrica y cuáles las propuestas de cambio planteadas en torno a 1775, cuando se cimentaron los muros de cerramiento de la fachada a poniente y gracias a estos planos entendemos cuánto dicha cimentación fue en realidad una argucia legal de la Diputación (frente al deseo del Ayuntamiento de enajenar el mismo para dignificar el trazado del camino Real) para mantener íntegra la propiedad.

Entre 1771 y 1788, momento en el que se suceden los pleitos entre Corporación Municipal y Seminario por el trazado del camino, la intención del Ayuntamiento era transformar el entorno de la ciudad mientras que el Seminario se oponía a tal trazado, reclamaba la propiedad de unos terrenos que nunca, hasta el momento había edificado. Urbanísticamente, conviene saber cuál fue la conclusión de aquellos pleitos: si la propuesta del Seminario era hacer pasar el Camino por delante del Colegio, orilla del río y "La Longuera", haza de la ciudad entre el convento de San Diego y el Seminario, el pleito, que se falló en 1775 favorablemente para el Colegio, aprobó hacer pasar el camino entre San Telmo y la Fábrica de Tabacos (construyéndose un foso algo "mas recogido" para no tocar los cimientos del Colegio) y luego continuar el camino pegado al foso, hasta San Diego, ordenando al Ayuntamiento a compensar al Colegio (como diferencia del valor por las tierras tomadas al camino) con "La Longuera" y 8.428 reales. Solventada la cuestión con el Cabildo de Sevilla, arquitectónicamente el problema se volvió a plantear cuando la Diputación pidió continuar las obras en un edificio que ahora se situaba inmediato a la Fábrica de Tabacos.

Al margen de que sucediera en el citado pleito, conviene destacar que si el Seminario no había construido antes en lo que evidentemente eran su propiedad es porque no precisaba de más espacio para sus instalaciones, porque lo existente bastaba y sobraba para la actividad que realizaba. Y esta cuestión, clave para comprender el final de las obras, obliga a que aceptemos el hecho de que estos planos nada dicen sobre cómo se organizaba la vida en el interior del Colegio, aspecto clave para entender la marcha de las obras, en los finales del XVIII, y cuyo conocimiento nos permite abordar la historia del edificio desde el conocimiento de su uso. 


\section{$068-069$ Criterios}

Transformaciones y cambios de San Telmo durante la segunda mitad del siglo XVIII

\section{PH51 - Diciembre 2004}

Entre 1770 y 1790 el Seminario vivió los avatares de los pleitos y los arquitectos (ingenieros militares, maestros de obras o académicos de San Fernando) se sucedieron sin que sepamos, a ciencia cierta, cuál fue la aportación real de cada uno: tenemos noticias de una primera intervención de Juan Téllez, José de Herrera, Antonio Camargo y Sabino Gutiérrez, supervisados todos por Antonio de Figueroa, y pocos años más tarde los nombres de Lucas Cintora, Ignacio Moreno, San Martín, Téllez o Camargo se solapan en los documentos que hacen referencia a la obra. Si los primeros fueron entalladores o maestros de obras, de los segundos conocemos su labor arquitectónica gracias a la documentación de la Academia de San Fernando. De algunos, como por ejemplo de Ignacio Moreno (arquitecto afincado en Sevilla hasta que en 1788 fue nombrado arquitecto en Baeza) tenemos noticias sueltas; de otros, como Cintora, sería necesario elaborar un estudio más detallado.

Ignacio Moreno aparece en la obra de San Telmo en los últimos momentos de la década de los setenta y primeros de los ochenta. Colaborador de Lucas Cintora (junto con San Martín y Roales) en el proyecto de Cuartel de Infantería presentado en Sevilla en 1776, gracias a los datos de la Comisión de Arquitectura de la Academia de San Fernando tenemos noticia que presentó para su aprobación propuestas tales como el Puente de Nájera y un cuartel de caballería que, junto con San Martín, traza para Sevilla. De Lucas Cintora los datos son más numerosos y, estudiados estos, resulta difícil valorarle (como hiciera Corbacho y asumiera Falcón, influidos quizá por la nota que brinda Llaguno sobre su actividad) como "arquitecto de prestigio". De "renombre", quizá; de "prestigio", entiendo que no. Señalo esto por las críticas que reiteradamente hizo la citada Comisión de Arquitectura de la Academia de San Fernando a los proyectos presentados: así, por ejemplo, cuando en agosto de 1786 pidió la aprobación de un trazado en Castillo de las Guardas (en colaboración con Echamorro), la Academia lo rechazó y censuró, no sólo por su mala distribución sino por su mal gusto, proponiendo en su lugar a Manuel Machuca. Igual cabría decir del rechazo que provocó su idea de matadero en Osuna, los dibujos de fuente pública en Montellano o las trazas para el Ayuntamiento Higueras, en Alacena, que obligaron a la Academia a proponer en su lugar a Ignacio Tomas. Por estos hechos se hace extraño pensar que Moreno o Cintora (profesionales sin formación académica y ajenos, en consecuencia, a las soluciones sobre organización de viviendas discutidas en los años ochenta en la Academia de Madrid) pudieran ser los autores de algunas de las soluciones presentadas, en 1783, para San Telmo, edificio del que, para conocer su realidad en dicho año es preciso recurrir al plano firmado por Francisco Pizarro.

En 1780 el Consejo de Indias encargó a Camargo la elaboración de cuatro planos sobre el edificio, reseñando lo existente y, sobre todo, formulando una propuesta de cómo concluir la fábrica. Sin duda Camargo desarrolló tal cometido y un año más tarde se pedía a Francisco Pizarro copia de aquellos planos. Lo que ha llegado hasta nosotros (conservado en el Laboratorio de Arte de la Universidad de
3 y 4. Cabecera de la capilla de San Telmo de L. de Figueroa y descendientes. Fotos Eugenio Fernández Ruiz y José Manuel Santos Madrid

Sevilla) es la planta baja del edificio donde, con detalle, no sólo se informa sobre la situación en que se encontraba la parte más antigua del Seminario (la que resulta ser la más compleja), no sólo se proponen pequeños cambios en la misma sino que, y sobre todo, se propone gráficamente cómo construir la parte no edificada, la zona que Figueroa reclamara en su propuesta.

Lo primero que se advierte al estudiar la planta del edificio que refleja el Plano de 1783 (al margen que su autor fuese Camargo, que lo trazase Moreno o que Pizarro fuera sólo un simple tracista) es que en el mismo existen tres partes claramente diferenciadas. Una primera, la más antigua y donde en esos momentos se centraban la totalidad de las actividades del Colegio, se encontraba entre el eje de Figueroa y el jardín que mira al río: una segunda zona correspondería a la definida por el eje definido por la Portada, Claustro e Iglesia; y una tercera, inexistente pero limitada en su perímetro por los muros de cimentación dispuestos con motivo de los pleitos, corresponde a la zona comprendia entre el citado eje la fachada que da a Puerta Nueva y Puerta de Jerez.

La Planta de 1783 informa, en la parte antigua del edificio, de cuál era el uso de las distintas estancias. Si bien el lamentable estado de conservación del documento impide conocer dónde se situaban algunas de ellas (la cartela del plano define su existencia, pero es difícil situarlas a veces con precisión) sin embargo es factible ubicar dónde se encontraban muchas otras definidas como salas, dormitorios, aulas, patios, viviendas... Estudiando su situación y buscando comprender la lógica de su organización interna, tras estudiar la planta del edificio creo poder destacar siete núcleos en torno a cada uno de ellos se definen programas de necesidades precisos. Independientes los unos de los otros, ocupan el espacio construido entre el patio y la fachada al río y es fácil señalar cómo, en el trazado de lo edificado, la voluntad por definir un edificio rectangular en planta (en el que se situaron y debieron construirse los citados siete "programas") primó sobre la voluntad por componer en planta y luego proyectar la fachada.

El primero de los núcleos o "programas de necesidades" corresponde a la zona de dormitorios para alumnos; un segundo se destina a Enfermería; otro, a cocina del Colegio; un cuarto, a Refectorio; un quinto, a casa del maestro de primeras letras; un sexto espacio, a escuela de primeras letras y un último es el destinado a Escuela del Seminario. El primero de los espacios así definidos (el que corresponde a los dormitorios) se articula en base a una doble crujía, en ángulo recto: si el dormitorio norte tiene unas dimensiones aproximadas de casi $76 \mathrm{~m}$. de longitud por 13,5 de ancho, dividido por 13 pies derechos (Io que presumiblemente da un total aproximado de 34 camas por frente -68 en total-) el dormitorio a levante, de igual ancho, era ligeramente más largo (dividido por 15 pies derechos, entiendo que su longitud se aproximaba a los noventa metros, con un total de 80 camas en ambos frentes). Ambas piezas tenían varios accesos: tres en la parte superior, que posibilitaban el paso hacia los patios porticados situados en el norte del edificio y uno más, que comunicaba con la Enfermería; en el dor- 


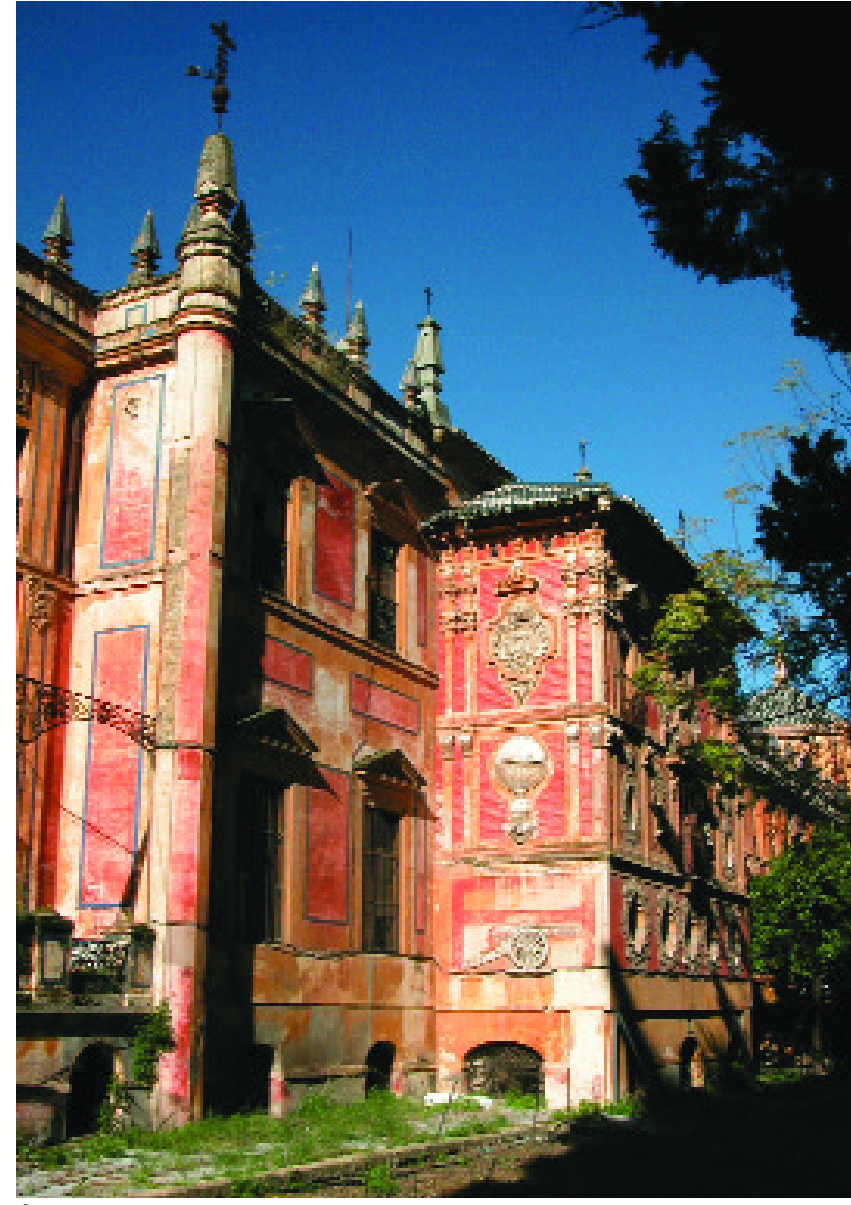

mitorio a levante los accesos eran, bien el gran acceso al dormitorio desde el pasillo que le unía con el Claustro, bien salidas a pequeños patios de servicio, bien -en la parte inferior- a las aulas, bien otro más que posibilitaba salir a los jardines inmediatos al río.

Inmediato al dormitorio a norte y próxima a la Iglesia se encontraba la Enfermería. Núcleo cuadrado, articulado en torno a patio, las cuatro salas para enfermos existentes se organizaban en torno a un pequeño patio al que comunicaba la cocina y lugar común (retretes), ventilados estos además por un patinejo. Entre Cocina y Refectorio se estableció el tercer programa, el correspondiente a la Cocina del Seminario donde, en torno a un patio central, se dispusieron tanto las despensas como los fogones, fregaderos... Comunicada directamente (en el extremo superior derecho) con el Claustro, contaba con un torno que permitía servir al Refectorio.

El cuarto programa, el Refectorio del Seminario, consistía en una gran sala de cuarenta metros de longitud que, situada estratégicamente en el centro de esta parte del edificio, comunicaba a derecha e izquierda tanto con la Escuela de Primeras Letras como con la Casa del Maestro, con el Pasillo Central y con la cocina. Valorado como espacio central del antiguo Seminario, entre el corredor principal del edificio y el acceso al Refectorio se dispuso un pequeño espacio casi cuadrado (de unos ocho metros de largo por el ancho de la crujía del Refectorio) concebido como espacio previo (antesala, cabria decir) al Refectorio. La Sala se iluminaba gracias al patio existente entre esta y la Escuela de Primeras Letras.

El quinto programa de necesidades definido en esta parte del edificio lo constituía la Casa del Maestro de Primeras Letras. Comunicado tanto con el Claustro como con el Refectorio, las habitaciones de esta parte se articulaban en torno a un pequeño patio (que con luz de poniente iluminaba, así mismo, el Refectorio) inmediato a unas escaleras que conducian a la parte superior, espacio donde sin duda residian empleados subalternos del Seminario dedicados a la educación de los escolares. La Escuela de Primeras Letras, compuesta por distintas habitaciones o aulas, contaba así mismo con dos patios de luz (zona de recreo quizá de los

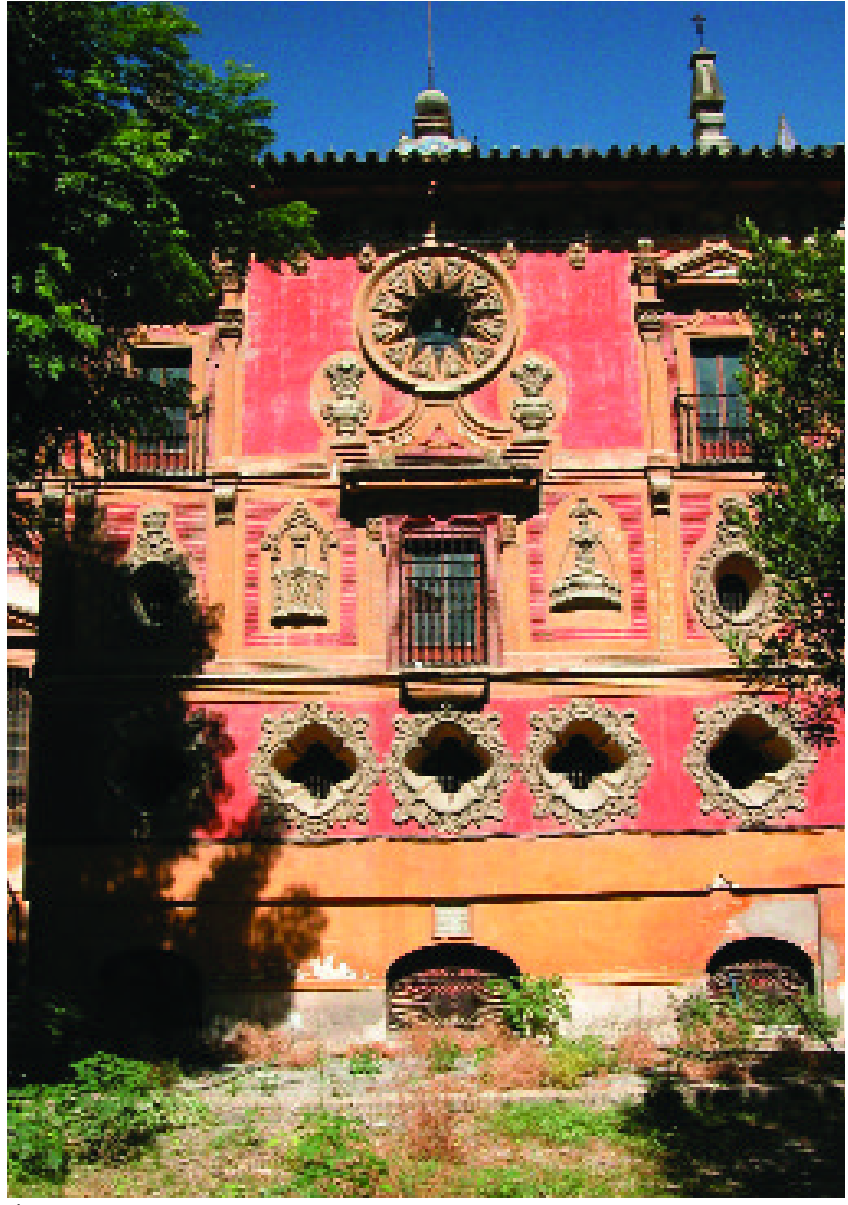

escolares) al tiempo que permitía el acceso a un gran patio cubierto comprendido, en la zona próxima a la fachada principal, entre el corredor y el dormitorio a levante. Por último, y dispuestas todas ellas dando a la fachada principal, se encontraban las aulas del Seminario.

Entiendo, como he señalado anteriormente, que la voluntad por definir un edificio rectangular en planta primó sobre la voluntad por componer en planta y definir luego la fachada. Los citados siete núcleos (cada uno con programa de necesidades propio) tuvieron que adecuarse al espacio dado. Si bien resulta dificil reconstruir el proceso de proyectación de esta parte es evidente que la organización interior se concibió desde una premisa clara: las dos salas destinadas a dormitorio debían ser fachadas y no podían dar a la fachada principal, entendiendo que las aulas era la "parte noble" del proyecto y que a estas correspondian dar al frente de fachada. Construido en consecuencia un primer bloque en forma de " $\mathrm{C}$ " invertida (tres crujías, dos correspondientes a los dormitorios y la tercera a las aulas del seminario) el problema fue articular el conjunto. Por ello, al estudiar cada uno de los siete programas citados podemos advertir cómo su singularidad radica no en que fueran independientes entre sí sino en el hecho (característico en los conventos sevillanos de la época) que todos se ordenaron en torno a un patio. Cada uno de los "programas" dispuso del suyo propio, cuando no de dos y, a su vez, cada patio tuvo dimensiones precisas en función del programa, del mismo modo que unos se concibieron como patios cubiertos y otros no. Hubo en el edificio, como refleja la Planta de Pizarro, patios comunes a varios "programas" (el patio central, concebido como charnela articuladora con la parte todavía no construida; el patio cubierto que se dispuso junto a la Escuela de primeras letras; el inmediato al testero de la Iglesia, con acceso al jardín y a la Enfermería...) del mismo modo que los patios "privados" solamente eran accesibles desde las habitaciones que configuraron cada una de estas partes, concebido para ser utilizado sólo por quienes viven o trabajan en su entorno. Y demostrando cuánto cada uno se había concebido para un espacio propio, cada uno de ellos, como refleja la documentación encontrada en el Archivo del palacio Real, disponía de fuente propia. 


\section{$070-071$ Criterios}

Transformaciones y cambios de San Telmo durante la segunda mitad del siglo XVIII

\section{PH51 - Diciembre 2004}

Construido, decía en el párrafo anterior, un primer bloque en forma de " $\mathrm{C}$ " invertida (tres crujías, dos correspondientes a los dormitorios y la tercera a las aulas del seminario) el problema fue articular el conjunto. En este sentido, al estudiar la planta de dicho conjunto sorprende lo que en un primer momento parece desorden compositivo: analizando con atención, se advierte la existencia de un doble eje (en "T" invertida) en torno al cual se articulan y disponen los diferentes proyectos. Si en el brazo paralelo al eje principal (dispuesto en el centro del conjunto) se sitúa el Refectorio, que comunica tanto con cocinas como con dormitorios, con la Casa del maestro o con la Enfermería, el brazo de la citada cruz paralelo a la fachada se concibe como corredor que permite atraviesa de un extremo a otro toda la zona, comunicando el Patio con las aulas del Seminario, el Refectorio y, por ultimo, con los dormitorios. Dicho de otra forma, mientras que los espacios destinados a la vida cotidiana del Seminario se agrupan en torno al eje que define el Refectorio, las zonas ligadas a la Universidad de Mareantes se ordenan en torno a este segundo brazo de la "T".

El plano que conocemos de Pizarro sólo es de planta baja: pero la disposición de las pequeñas escaleras (que, como los patios, se encuentran en los siete "programas" señalados, imagino que para uso y disfrute exclusivo de quien utiliza el mismo) permite intuir cuánto la organización de la planta superior no tuvo que ser muy distinta a la que señala la planta baja. Por otra parte, la falta de una sección dificulta entender cómo se organizó la trasera del edificio, máxime cuando en el plano de 1775 se había señalado como San Telmo era ...una edificación de tres fachadas. Porque tras la crujía norte de los dormitorios aparecen un conjunto de espacios (de utilización confusa, al describirse en la cartela del plano como "patios para las diferentes partes") de utilización exclusiva de los colegiales, por su inmediatez a los dormitorios. Pero no es este conjunto el único que desconcierta, porque en el mismo documento se propone, paralelo a la crujía de la Capilla y como pieza a la que da singular importancia, una larga crujía de la que nada sabemos sobre su utilización (el plano está dañado en ese punto) y que no sólo se concibe como clave en el desarrollo norte del edificio sino que rompe la idea de planta rectangular que desde el primer momento tuviera el edificio. Pero lo más sorprendente del plano de Pizarro es la información que facilita sobre la parte todavía no construida y la ordenación que hace de aquel espacio.

Cuando en 1783 Pizarro propuso, en su proyecto, edificar la parte todavía no construida, sugirió modificaciones y cambios en zona comentada. Bien es cierto que estos fueron mínimos y conviene destacar cómo en nada cambiaban el orden existente. Es importante recalcar este aspecto porque demuestra cuánto un arquitecto de cultura ilustrada y reformista (como se advierte en su propuesta de intervención para la parte no construida del edificio) era sin embargo capaz de aceptar un programa barroco, al entender que tal forma de disponer los espacios y resolver los problemas fue característica al barroquismo sevillano. Sin embargo, en la parte no edificada la propuesta se planteó desde un saber hacer radicalmente distinto al existente en la parte anterior, reflejo de cómo a
Io largo del XVIII hubo dos formas distintas de entender y concebir la arquitectura.

Al estudiar la planta de la obra a concluir sorprende, en primer lugar, ver el papel fundamental que adquiere un gran patio central, en torno al cual se disponen los distintos espacios. Inexistente en la parte construida (sustituye, por así decir, el corredor que unía el patio con el Refectorio y dormitorios) importa destacar cómo su presencia demuestra como la historia de la arquitectura para nada debe valorarse como "historia de los estilos" o de los "lenguajes" (la historia de la arquitectura no debe ser un "cambio de estilos" - la interpretación sobre distintos "lenguajes clasicistas") y si el saber que permite valorar y comprender que supone la sustitución de un pasillo por un patio (que no patinejo, como sucede en los siete programas señalados) cuya función es articular y definir lo que ahora se proyecta.

En segundo lugar, sorprende, al ver la zona que vierte tanto a la panda norte como a la de poniente del citado patio, lo que se define como "casas de maestros" y que resulta de proyectar (dentro del proyecto) cuanto menos cinco viviendas independientes. De alguna manera, cabría señalar, el autor de la propuesta quiso dejar la parte antigua del edificio a los escolares y destinar la nueva a otros servicios. Las cinco viviendas proyectadas ocupan casi íntegramente lo que resta de fachada norte (entre la capilla y el torreón situado a su izquierda) así como casi en su totalidad el frente de la fachada a poniente: es decir, se busca concluir el edificio... sólo para construir viviendas para los maestros y no porque exista un programa claro y especifico de necesidades.

En el plano de 1783, se puede comprobar cuánto la Diputación del Seminario carecia de programa de necesidades para aquella zona y la documentación gráfica que ha llegado hasta nosotros, nada precisa sobre qué debía haberse edificado en la misma. La propuesta para la nueva obra consistió sólo en dar forma a un impreciso programa de viviendas para los maestros, dispuestas en torno a un patio, donde la forma de adosar unas y otras (la ordenación en planta de viviendas) demuestra un saber hacer característico de la segunda mitad del XVIII, ajeno ya a disposición en planta de los últimos años del XVII. Lo cual, en buena lógica, nos lleva a suponer cuánto la propuesta de Pizarro (o de quien fuera su autor) fue más una reivindicación de un suelo amenazado por las reformas de Olavide que la voluntad real de concluir unas obras necesarias.

Durante toda la mitad del XVIII existe en consecuencia un singular debate entre quienes entienden que la forma rectangular de la planta debía ser determinante para el desarrollo de la propuesta y quienes, desde la crisis económica existente, se niegan a obras no necesarias, actuando sólo cuando la amenaza del Ayuntamiento en ocupar el terreno no edificado (con objeto de hacer pasar a través del mismo el Camino) lleva a debates y pleitos donde los planos propuestos son más coartadas utilizadas en pleitos que no propuestas reales de obra. Por ello, la importancia y el interés del plano de 1783 de Francisco Pizarro. 